\title{
Duration of Jovian magnetospheric disturbances inferred from decametric radio storms
}

\author{
Akira Morioka ${ }^{1}$, Fuminori Tsuchiya ${ }^{1}$, Yoshizumi Miyoshi ${ }^{1}$, Hiroaki Misawa ${ }^{1}$, Hiroshi Oya ${ }^{2}$, and Kinji Furukawa ${ }^{3}$ \\ ${ }^{1}$ Planetary Plasma and Atmospheric Research Center, Tohoku University, Sendai 980-8578, Japan \\ ${ }^{2}$ Department of Space Science and Communication, Fukui University of Technology, Fukui 910-8505, Japan \\ ${ }^{3}$ National Space Development Agency of Japan, Tokyo 105-8060, Japan
}

(Received May 7, 2002; Revised xxxx xx, 2002; Accepted November 21, 2002)

\begin{abstract}
We investigated the persistence of the most intense Jovian decameter bursts observed during 17 consecutive years (1974-1990). The results showed that even the most intense group of decametric storms lasted only oneearth-day or less. When we assume that the persistence of the Jovian decametric radio storms indicate the duration of the Jovian magnetospheric disturbance, the result implies that even the large Jovian magnetospheric disturbance appears in a major singular event without sequential activities. From this argument, it would be supposed that the Jovian magnetosphere unloads the stored magnetospheric energy in a burst and has no geomagnetic storm-like disturbance.
\end{abstract}

\section{Introduction}

The Jovian magnetosphere is fundamentally driven not by the solar wind but by its own fast rotation. The major energy source of the Jovian magnetospheric plasma is considered to be the predominant planetary rotation. This is the essential difference between the Jovian and terrestrial magnetospheres. However, in case of the Jovian magnetosphere little is known concerning the storage and release processes of energy and the functions of the solar wind disturbances. Therefore great interest has focused on whether the Jovian magnetosphere has the disturbances with the bursty energy release process as in the case of the earth, and how the rotationally driven giant magnetosphere responds to the solar wind disturbances.

The Galileo observations revealed large scale magnetospheric disturbances, and some evidences have been presented claiming the existence of the storm and/or substormlike phenomena in the Jovian magnetosphere. Mauk et al. (1997, 1999) reported the storm-like plasma injection phenomenon in the Jovian inner and middle magnetospheres and discussed their similarities with those of the earth's magnetospheric events. Woch et al. (1999) showed an event that exhibited dynamic process in the magnetotail and argued some analogies to the earth's substorm development. The quasiperiodic modulations of the Jovian magnetotail (Woch et al., 1998) and related bursty tailward plasma flows (Krupp et al., 1998) were also reported based on the Galileo observations. Louarn et al. (1998, 2000) showed the large scale energetic events in the Jovian magnetosphere. These important observations in the magnetosphere, however, could not show exactly whether the event is internally driven or stimulated by the solar wind as in the case of the earth's storm and/or

Copy right $(\mathrm{C}$ The Society of Geomagnetism and Earth, Planetary and Space Sciences (SGEPSS); The Seismological Society of Japan; The Volcanological Society of Japan; The Geodetic Society of Japan; The Japanese Society for Planetary Sciences. substorm.

On the other hand, remote observations can give information, although indirect, on both global magnetospheric events and the solar wind disturbances. Recently, Waite et al. (2001) reported a very intense auroral flare event in the Jovian polar cap region which might be related to solar wind shock. The association of the solar wind to the Jovian magnetosphere has been discussed using remote observations of Decametric Radio Wave (DAM). The relation of DAM occurrence with the solar wind variation was first investigated using ground based observations (e.g. Carr et al., 1960; Barrow, 1972; Oya and Morioka, 1981), and it was shown that occurrence of DAM has a positive correlation with the solar wind variation. Later, it was reported that not only DAM but also Jovian hectometric (HOM) and kilometric (KOM) radio waves are also correlated with the solar wind (e.g. Desch and Barrow, 1984; Barrow et al., 1988; Reiner et al., 2000). These evidences mean that the solar wind variation triggers and/or enhances the activity of the Jovian magnetosphere, because DAM and HOM are considered to be generated in the polar magnetosphere as the result of the magnetospheric particle acceleration. Menietti et al. (1999) investigated the local time dependence of Jovian radio emissions using the Galileo observation, and showed that DAM is more intense and more frequent in the midnight sector. They argued that DAM may indicate the existence of Jovian substorm controlled by the solar wind because the occurrence characteristics are in analogy to earth's substorm. Recently, the solar wind control of the Jovian radio emission and aurorae was clearly reported from the simultaneous observations using the Cassini and Galileo spacecraft (Gurnett et al., 2002). Studies on the direct relation between the Jovian particle phenomena and the solar wind variations have shown that the solar wind controls the energetic particle release from the Jovian magnetosphere (Morioka and Tsuchiya, 1996; Tsuchiya et al., 1999). From these remote 
observations of the Jovian magnetospheric phenomena, it is suggested that some magnetospheric disturbances might be controlled by the solar wind.

The energy loading and unloading process in the Jovian magnetosphere is one of the current topics on the standpoint of the comparative planetary magnetosphere. To understand the energy unloading process in the Jovian magnetosphere, it would be necessary to investigate the time scale of these Jovian disturbances and compare its process with that of the earth's storm/substorm.

In this paper, we considered that the duration of the magnetospheric disturbance would have information on the unloading process, and the duration can be derived from the persistence of huge DAM events which would be the manifestation of the distinct magnetospheric disturbance. The persistence of the anomalously intense DAM storms is studied to derive the upper limit of the duration for the energy release at the time of the Jovian magnetospheric disturbance.

\section{Large Decametric Radio Wave (DAM) Storms}

The top panel of Fig. 1 shows the earth's Dst index during the period from October 1, 1989 to January 31, 1990. In this period, there were two large magnetic storms with Dst indices exceeding $-250 \mathrm{nT}$, on October 20 and November 17, respectively. These storms belong to the largest class of disturbances during the last solar cycle. The middle panel of Fig. 1 shows the DAM activity chart which is the daily plot of the power index of the Jovian DAMs observed on the ground (Zao Observatory of Tohoku University). The power index (relative power) of each DAM event was determined by the product of the average amplitude of the DAM and the duration of the event. The DAM activities exceeding more than 400 of the power index are extremely large and rare events as will be shown later. In the figure, the DAM activity chart showed the occurrence of two anomalously large radio wave storms on October 29 and November 30 with about 9 and 13 days delays with respect to the earth's geomagnetic storms. This time delayed relation between earth's magnetic storms and the Jovian decametric storms suggests that the fast solar wind disturbances with the average velocity of about $770 \mathrm{~km} / \mathrm{s}$ (on October 20) and $550 \mathrm{~km} / \mathrm{s}$ (on November 17) first blew out around the earth causing large geomagnetic storms, and then hit the Jovian magnetosphere after traveling in interplanetary space and caused huge decametric radio wave storms. Considering the geometrical positions among the sun, earth and Jupiter, the conditions of the DAM storm on November 30 are illustrated in the bottom panel of Fig. 1, where the solar wind with the average velocity of $550 \mathrm{~km} / \mathrm{s}$ had passed through the earth on November 17 and reached Jupiter on November 30.

The present analysis that showed clear coincidence between the geomagnetic storm and Jovian huge DAM storm is basically consistent with the past studies (Carr et al., 1960; Barrow, 1978; Oya and Morioka, 1981) regarding the correlation of DAM with the solar wind variation. Based on these observations, we can suppose inductively that the huge DAM storms represent the occurrence of large Jovian magnetospheric disturbances triggered by the severe solar wind disturbances.
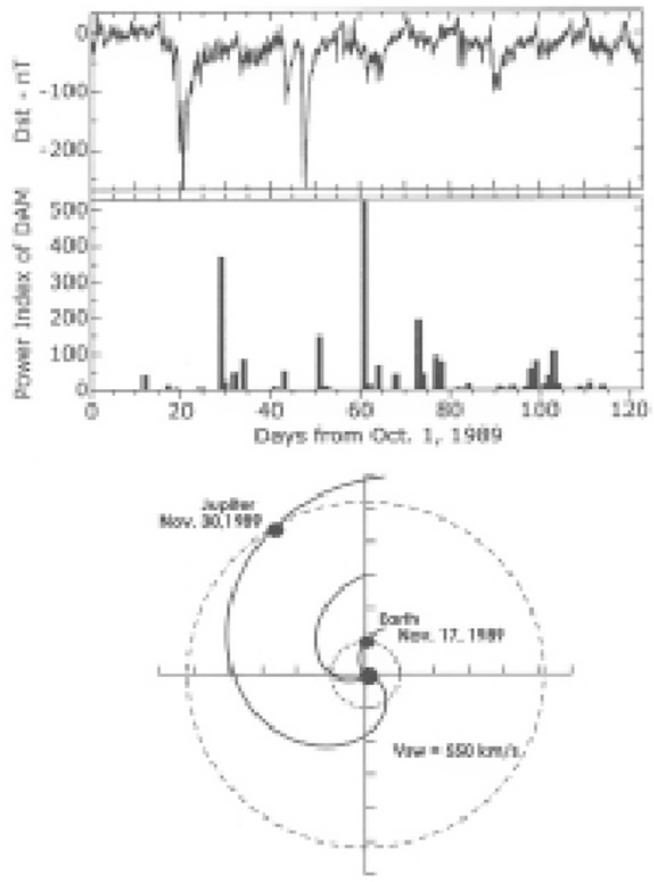

Fig. 1. Top: Dst index from October 1, 1989 to January 31, 1990. Two large magnetic storms occurred with Dst indices exceeding $-250 \mathrm{nT}$. Middle: The activity chart of the Jovian decametric radiations (DAM). Two huge DAM storms were detected with delays of 9 and 13 days with respect to the earth's two storms, respectively. Bottom: The inferred propagation of the fast solar wind disturbance with the velocity of 550 $\mathrm{km} / \mathrm{s}$ in interplanetary space at the time of the second DAM storm.

\section{Persistence of Large DAM Storms}

In the case of geomagnetic storm, radio emissions and related wave phenomena, such as auroral kilometric radiation (as will be shown in the next section) and VLF emission (Kasahara, private communication), show the intense activity during the storm. These radio phenomena should be the manifestation of the energy loss process of the magnetospheric disturbances injected into the polar magnetosphere, and continue during the magnetospheric disturbance.

When we consider that huge DAM events are the indication of the Jovian magnetospheric disturbances on the bases of the comparative planetary radio wave phenomena, it would be able to derive information on the characteristic time of the energy unloading process in the Jovian magnetosphere, using the persistence of DAM storms.

From this point of view, the persistence of huge DAM storms was investigated using the DAM database provided by Tohoku University since 1974 (Oya and Morioka, 1979). The analyzed period was from October 1, 1974 to December 31, 1990. Only non-Io related sources of DAM were considered since these sources reflect the effect of the solar wind (Barrow, 1978), while Io-related DAM sources reflect a different energy from Io in the inner magnetosphere. The total number of the non-Io related DAM events was 551 during the study period (17 years), and 27 of the largest DAM events $(4.9 \%)$ with the power index values of more than 400 were selected as huge DAM storms. Medium and small DAM events might not be always generated through distinct stormlike disturbances but through localized or small disturbances. 
On the other hand, large DAM events are considered to be generated through the global magnetospheric disturbances triggered by the large solar wind variations as presented in the previous section and would reflect the typical response to the large magnetospheric disturbances, providing the upper limit of the phenomenal characteristics. For this reason, we selected the large intensity DAM group for this study.

To investigate the persistence and decay time of the huge DAM storms, the time profile of daily DAM power index for the period of 10 days before and after the commencement of the huge DAM storms, was plotted. Figure 2 shows examples of the plots, where the main events are set at the center of the plots. The events on October 4, 1987 (upper panel) and on December 12, 1987 (lower panel) have the power index of 833 and 910 (the most intense one during the analyzed period), respectively. The power index distribution of all 551 events is shown in the right side of each panel, and the arrow indicates the index of the interested event. In the figure, the huge DAM emission was observed on the event day ( 0 day in the figure), and on the next day, a small DAM emission that has common intensity was detected. This shows clearly that a huge DAM event occurs sporadically, and persists for only one day, without any specific activity that precedes or follows the main event. This evidence is rather surprising because the vast Jovian magnetosphere is simply supposed to need some reaction time and to have a longer inertia time to release the large volume of stored energy in the plasma sheet and/or plasma disc. Here, it should be noticed that we used daily averaged DAM intensity in this study, so that the real persistence of the DAM storm might be less than one day.

Next, a normalized super epoch analysis was applied for all 27 huge DAM events to investigate the statistical feature of DAM persistence. The peak power values of the 27 events were normalized by the power of the main event at $t=0$ day, and power values for each day were averaged after adopting the normalization. The result is shown in Fig. 3. It is also statistically concluded that the persistence of a huge DAM storm event lasted only one day or less, suggesting that the Jovian magnetosphere has no long lasting storm-like disturbances unlike the earth's magnetic storm that takes 2 or 3 days to recover the pre-storm state.

\section{Comparison with the Earth's AKR}

AKR, a good indicator of the earth's magnetospheric disturbances (Gurnett, 1974; Voots et al., 1977), might be a counter part of the Jovian decametric radiations. The relation of AKR with the terrestrial magnetic storm was investigated from the standpoint of comparative magnetospheric disturbances. As an example, a large magnetic storm commenced on November 17, 1989 (same that the first storm in Fig. 1) is shown in Fig. 4. Six dynamic spectra of AKR observed over the polar region by the Akebono satellite (Oya et al., 1990) are shown corresponding to the period during 1 day before and 5 days after the magnetic storm commencement. The AKR activity was quiet on the day before the storm. After the storm commencement on November 17, the AKR activity was strongly enhanced in the frequency range from about $150 \mathrm{kHz}$ to $400 \mathrm{kHz}$ and the enhanced AKR activity lasted, at least, for 4 days. Although AKR is essentially substorm related phenomenon, storm time AKR persists for several days

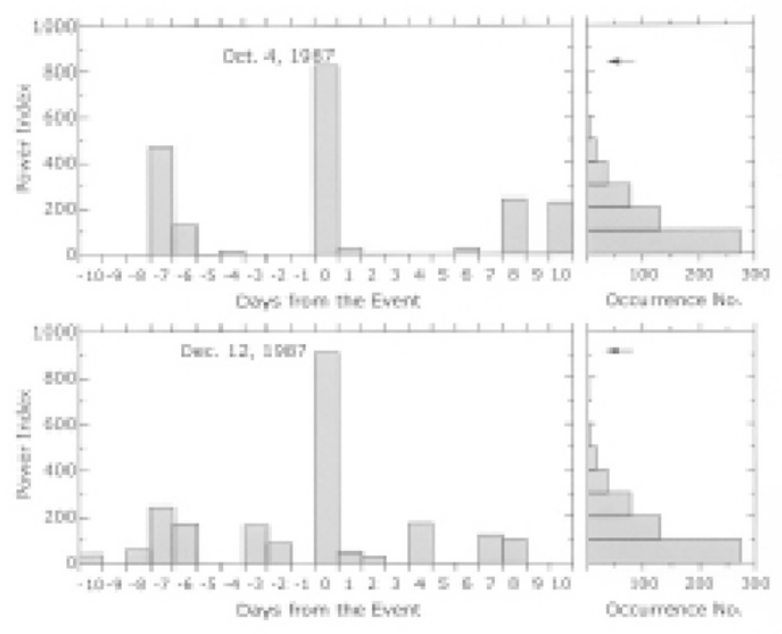

Fig. 2. Activity chart of huge DAM storms. Two huge DAM storms (October 4, 1987: upper, and December 12, 1987: lower) are shown. The huge DAM storms occurred sporadically and had no preceding and following activities. The power distribution of DAM events is in the right. The arrow shows the power index of the interested event.

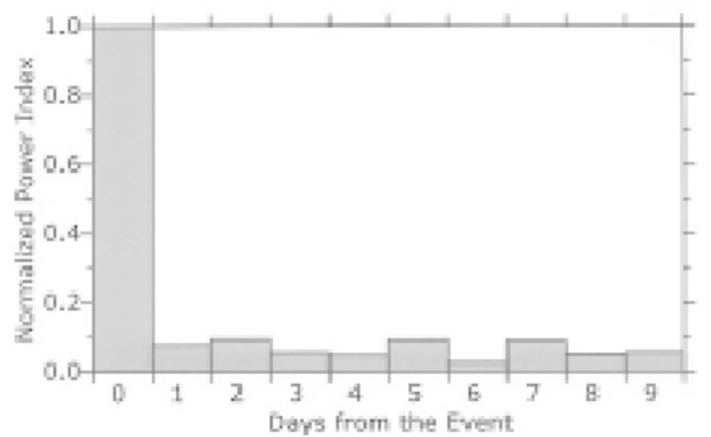

Fig. 3. The statistical power index profile after the on-set of the huge DAM storm by means of the normalized super epoch analysis.

indicating that a series of substorm takes place during the storm. This is quite different from the Jovian DAM phenomena which are always isolated without any sequential activities.

This difference between Jupiter and the earth was also statistically investigated. To study the statistical decay time of the storm-related AKR activities, 38 of isolated magnetic storms detected from 1989 to 1998 were selected. For the electric field data observed by Akebono during the selected storm periods, we defined the radio wave phenomenon as a AKR which has the typical AKR frequency band between 200 to $600 \mathrm{kHz}$, and is observed in the most probable occurrence region of AKR, where MLT is from 18 to 03 and $L$ value ranges from 4 to 7 , above the satellite altitude of 3000 $\mathrm{km}$. Based on these definitions, averaged daily AKR intensity was calculated. The super epoch analysis for Dst and AKR intensities of the 38 storms is shown in Fig. 5, where the day of the minimum Dst is referred as the epoch day. Compared with the result of Fig. 3, the result indicates that, in great contrast with that of the Jovian DAM storm, AKR activity continues for more than 4 days after the storm com- 


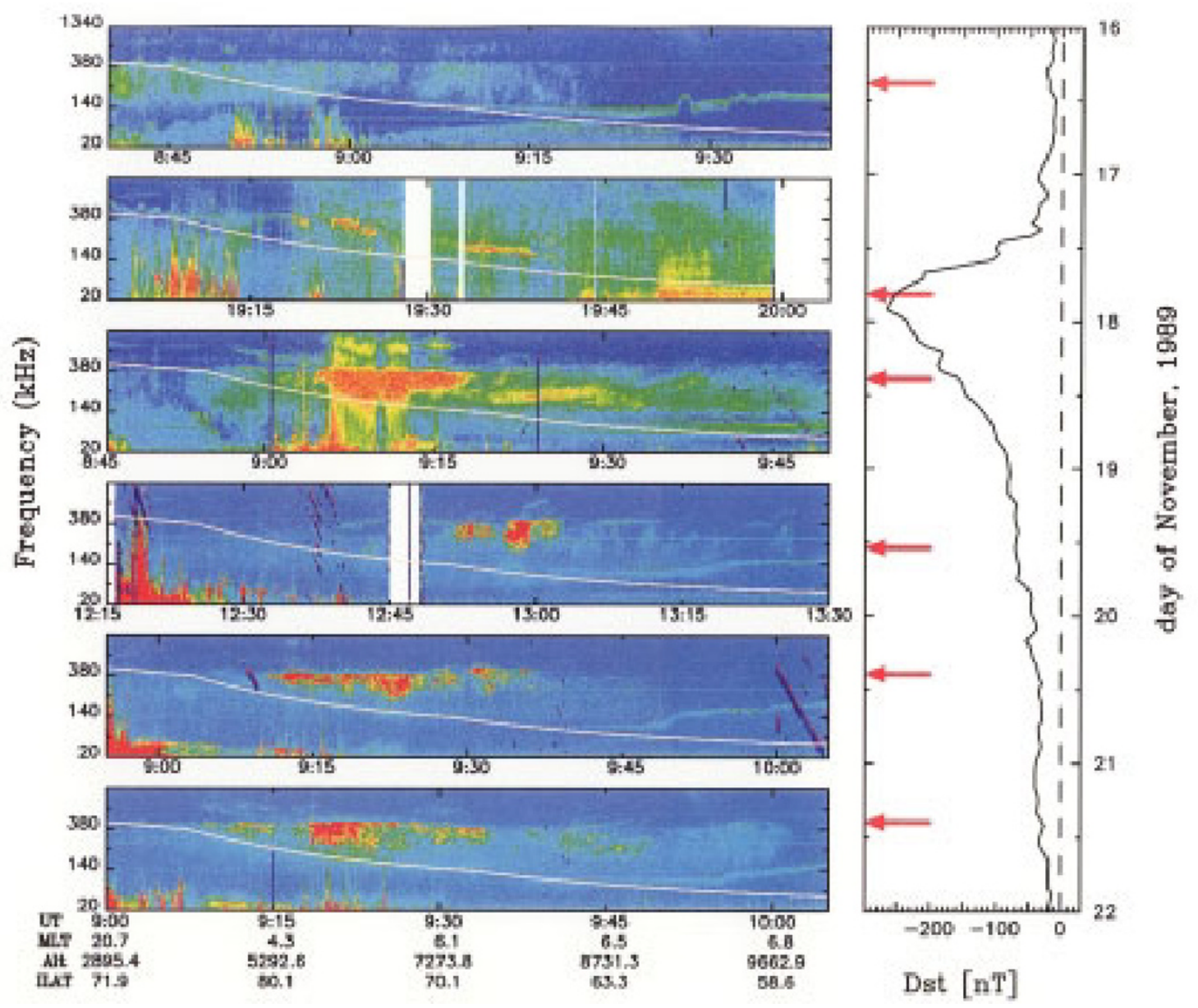

Fig. 4. Dst index (right) and dynamic spectra of AKR (left) observed in the polar region by the Akebono satellite at the time of the magnetic storm on November 17, 1989. White thin lines are the local electron cyclotron frequency. The orbital parameters of the satellite shown at the bottom are for the orbit on Nov. 21 (bottom dynamic spectrum). The orbital parameters for the preceding 5 days are not so different from those on Nov. 21 . The AKR activity in the frequency range from 150 to $400 \mathrm{kHz}$ was enhanced at the time of the storm commencement and lasted 4 days after the storm commencement.

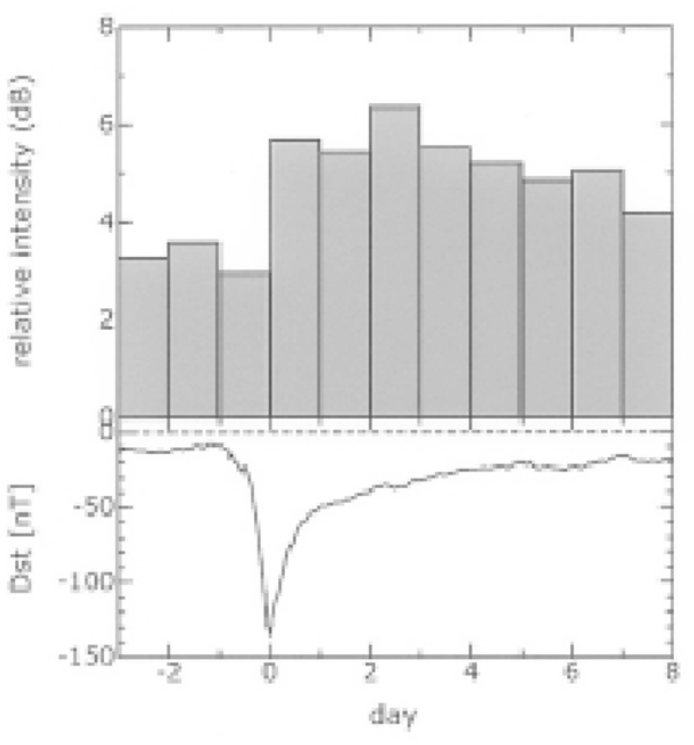

Fig. 5. The statistical time profiles of AKR power (upper) and Dst index (lower), which are superposed referring the day of the minimum Dst. mencement.

\section{Summary}

We confirmed through the typical case studies that large solar wind disturbances trigger the huge DAM events which should be resulted from the magnetospheric disturbances. This can allow us to regard the huge DAM events as the manifestation of the occurrence of large magnetospheric disturbances. It can be also supposed that, when a long-lasting large disturbance occurs in the Jovian magnetosphere, the huge DAM storm would be detected during the period of the disturbance. Under these considerations, we investigated how many days huge DAM events repeat (persist) corresponding to the large magnetospheric disturbance. The result showed that huge DAM storms last for only one earth day or less. Thus, we infer that the Jovian magnetospheric disturbances caused by the solar wind interaction would release the energy in one major singular event without any sequential disturbances. This implies that geomagnetic storm-like phenomenon, which releases the energy over several days, might not exist in the Jovian magnetosphere. We suppose that the giant and rotationally driven Jovian magnetosphere is a quick response system to unload the stored magneto- 
spheric energy.

As mentioned in the Introduction, spacecraft observations detected detailed in-situ magnetospheric processes, such as quasi-periodic modulations of the magnetotail (Woch et al., 1998), energetic particle bursts (Krupp et al., 1998) and 3days dynamical events (Louarn et al., 1998, 2000). These are obviously global and recurrent magnetospheric phenomena. Louarn et al. (2000) claimed that 3-day dynamical events are the result of the internal loading and unloading process that are common in the Jovian magnetosphere. On the other hand, large magnetospheric disturbances derived from huge DAM storm in this paper would be a different kind of large disturbances that are presumably related to the solar wind.

Acknowledgments. This work was supported by grant-in-aid for Scientific Research (12440129) from the Ministry of Education, Science, Sports and Culture, Japan.

\section{References}

Barrow, C. H., Decameter-wave radiation from Jupiter and solar activity, Planet. Space Sci., 20, 2051-2056, 1972.

Barrow, C. H., Jupiter's decametric emission and solar activity, Planet. Space Sci., 26, 1193-1199, 1978.

Barrow, C. H., Y. Leblanc, and M. D. Desch, The solar wind control of Jupiter's broad-band kilometric radio emission, Asrton. Astrophys., 192, 354-359, 1988

Carr, T. D., A. G. Smith, and H. Bollhagen, Evidence for the solar corpuscular origin of the decameter-wavelength radiation from Jupiter, Phys. Rev. Lett., 5, 418-420, 1960.

Desch, M. D. and C. H. Barrow, Direct evidence for solar wind control of Jupiter's hectometer-wavelength radio emission, J. Geophys. Res., 89, 6819-6823, 1984.

Gurnett, D. A., The earth as a radio source: Terrestrial kilometric radiation, J. Geophys. Res., 79, 4277-4238, 1974.

Gurnett, D. A., W. S. Kurth, G. B. Hospodarsky, A. M. Persoon, P. Zarka, A. Lecacheux, S. J. Bolton, M. D. Desch, W. M. Farrell, M. L. Kaiser, H.-P. Ladreiter, H. O. Rucker, P. Galopeau, P. Louarn, D. T. Young, W. R. Pryor, and M. K. Dougherty, Control of Jupiter's radio emission and aurorae by the solar wind, Nature, 415, 985-987, 2002.

Krupp, N., J. Woch, A. Lagg, B. Wilken, and S. Livi, Energetic particle bursts in the predawn Jovian magnetosphere, Geophys. Res. Lett., 25, 1249-1252, 1998.

Louarn, P., A. Roux, S. Perraut, W. S. Kurth, and D. A. Gurnett, A study of the large scale dynamics of the Jovian magnetosphere using the Galileo plasma wave experiment, Geophys. Res. Lett., 25, 2095-2098, 1998.
Louarn, P., A. Roux, S. Perraut, W. S. Kurth, and D. A. Gurnett, A study of the Jovian "energetic magnetospheric events" observed by Galileo: role in the radial plasma transport, J. Geophys. Res., 105, 13073-13088, 2000.

Mauk, B. H., D. J. Williams, and R. W. McEntire, Energy-time dispersed charged particle signatures of dynamic injections in Jupiter's inner magnetosphere, Geophys. Res. Lett., 24, 2949-2952, 1997.

Mauk, B. H., D. J. Williams, R. W. McEntire, K. K. Khurana, and J. G. Roederer, Storm-like dynamics of Jupiter's inner and middle magnetosphere, J. Geophys. Res., 104, 22,759-22,778, 1999.

Menietti, J. D., D. A. Gurnett, W. S. Kurth, and J. B. Groene, Local time dependence of Jovian radio emissions observed by Galileo, Geophys. Res. Lett., 26, 569-572, 1999.

Morioka, A. and F. Tsuchiya, Solar wind control of Jovian electron flux: Pioneer 11 analysis, Geophys. Res. Lett., 23, 2963-2966, 1996.

Oya, H. and A. Morioka, Development of observing station for Jovian decameter wave reception at Mt. Zao observatory of Tohoku University, J. Geomag. Geoelectr., 31, 47-66, 1979.

Oya, H. and A. Morioka, Relations between turbulent regions of interplanetary magnetic field and Jovian decametric radio wave emissions from the main source, Planet. Space Sci., 29, 783-791, 1981.

Oya, H., A. Morioka, K. Kobayashi, M. Iizima, T. Ono, H. Miyaoka, T. Okada, and T. Obara, Plasma wave observation and sounder experiments (PWS) using the Akebono (EXOS-D) satellite-Instrumentation and initial results including discovery of the high altitude equatorial plasma turbulence, J. Geomag. Geoelectr., 42, 411-442, 1990.

Reiner, M. A., K. L. Kaiser, and M. D. Desch, Long-term behavior of Jovian nKOM radio emission observed during the Ulysses-Jupiter encounter, Geophys. Res. Lett., 27, 297-300, 2000.

Tsuchiya, F., A. Morioka, and H. Misawa, Jovian electron modulations by the solar wind interaction with the magnetosphere, Earth Planets Space, 51, 987-996, 1999.

Voots, G. R., D. A. Gurnett, and S.-I. Akasofu, Auroral kilometric radiation as an indicator of auroral magnetic disturbances, J. Geophys. Res., 82, 2259-2266, 1977.

Waite, J. H., G. R. Gladstone, W. S. Lewis, R. Goldstein, D. J. McComas, P. Riley, R. J. Walker, P. Robertson, S. Desai, J. T. Clarke, and D. T. Young, An auroral flare at Jupiter, Nature, 410, 787-789, 2001.

Woch, J., N. Krupp, A. Lagg, B. Wilken, and S. Livi, Quasi-periodic modulations of the Jovian magnetotail, Geophys. Res. Lett., 25, 1253-1256, 1998.

Woch, J., N. Krupp, K. K. Khurana, M. G. Kivelson, A. Roux, S. Perraut, P. Louarn, A. Lagg, D. J. Williams, S. Livi, and B. Wilken, Plasma sheet dynamics in the Jovian magnetotail: Signatures for substorm-like processes?, Geophys. Res. Lett., 26, 2137-2140, 1999.

A. Morioka (e-mail: morioka@pparc.geophys.tohoku.ac.jp), F. Tsuchiya, Y. Miyoshi, H. Misawa, H. Oya, and K. Furukawa 